\title{
FUSING UNCERTAIN, IMPRECISE AND PARADOXIST INFORMATION (DSMT)
}

$\mathrm{T}$

The scientific area of sensor information fusion is one of the most significant, fundamental, and actively researched areas of information fusion. Generally, it has the advantage of: (1) using redundant information, (2) using the complementarities of the available information, (3) getting more reliable information, and (4) improving the decision-making process. Its main purpose is to gather, manipulate, and interpret the information accurately and efficiently at various levels of abstraction, which occur in the multi-sensor integration problem, due to the fact that the data are input at one level of abstraction (sensor-specific level) and displayed at another - generalized level of abstraction (nature/class/type). The overall aim is to ensure that the sensors remain in balance for as much of the time as possible, so that they are capable of detecting all possible changes in the problem under consideration. Without fusion, it is difficult to guarantee that our perception of a problem, in time, would remain accurate, adequate, and consistent.

In general, sensor data is inaccurate, incomplete and uncertain, or even paradoxical or/and conflicting. Imperfections pervade real-world scenarios and have to be incorporated into every information system that attempts to provide a complete and accurate model of the real world. The nature of uncertainty depends on the mathematical theory, within which problem situations are modeled. Any mathematical theory is capable of capturing only certain particular type/aspects of it. The classical mathematical approaches for characterizing situations under uncertainty are Probability theory, Dempster-Shafer evidential reasoning theory, Fuzzy set/logic theory. However, none of the models for imperfect data available to date can fit to all forms of imprecise information. These models are complementary, not concurrent.

The real challenge is in recognizing the nature of imprecision, uncertainty, conflicts and paradoxes encountered in the particular problem. This volume of Information \& Security: An International Journal is devoted to some new advances and applications of the Theory of Plausible and Paradoxical Reasoning (DSmT), developed by Dr. Jean Dezert (ONERA, France) and Prof. Florentin Smarandache (University of New Mexico, USA). It proposes a new flexible general approach to managing both uncertainty and conflicts/paradoxes for a wide class of static or dynamic fusion 
problems, where the information to combine is modeled as a finite set of belief functions provided by independent sources of information. Its important contribution consists in overcoming the practical limitations of the Dempster-Shafer theory, related to the acceptance of the law of the third excluded middle. DSmT works for any model, which fits adequately with the true nature of the fusion problem under consideration and that way can be interpreted as a general and direct extension of Probability theory and Dempster-Shafer theory.

The first part of this I\&S volume is oriented to some theoretical advances of the Theory of Plausible and Paradoxical Reasoning: an introduction of the important fusion of both quantitative and qualitative beliefs; a generalization of the classic combination rules to DSm hyper-power sets; and a new class of fusion rules based on T-Conorm and T-Norm fuzzy operators. The second part of this journal volume presents some interesting applications of DSm theory, including: ordered DSmT and its application to the definition of continuous DSm models; robot map building from sonar sensors and DSmT; and human expert fusion for image classification. This dual presentation makes this volume important and interesting for both theoretical and applied scientists.

Prof. Stoyan Markov, DSc. Institute for Parallel Processing Bulgarian Academy of Sciences Sofia, Bulgaria May, 2006 